\title{
In silico analysis identifies CRISP3 as a potential peripheral blood biomarker for multiple myeloma: From data modeling to validation with RT-PCR
}

\author{
DONG LENG ${ }^{1}$, RAN MIAO ${ }^{2}$, XIAOXI HUANG ${ }^{2}$ and YING WANG ${ }^{1}$ \\ ${ }^{1}$ Clinical Laboratory; ${ }^{2}$ Medical Research Center, Beijing Chao-Yang Hospital, \\ Capital Medical University, Beijing 100020, P.R. China
}

Received September 27, 2016; Accepted January 5, 2018

DOI: $10.3892 / 01.2018 .7969$

\begin{abstract}
Octamer-binding protein 2 (Oct2) binds to the ATGCAAAT octamer on the IgH enhancer and stimulates IgH expression in human multiple myeloma (MM). Cysteine-rich secreted protein 3 (CRISP3) possesses the ATGCAAAT sequence and thus is activated by Oct 2 in mouse B cells, suggesting that CRISP3 may be activated in and be a potential biomarker for MM. The present study involved a meta-analysis of the gene expression profiling data of human MM peripheral blood. Significantly expressed genes were analyzed on merged super array microarray data and selected sample data with significantly expressed genes were additionally analyzed by principal component analysis and Bayesian probit regression. CRISP3, Oct2, Apha-1B-glycoprotein (A1GB) and Cyclin D2 (CCND2) were validated in clinical MM peripheral blood samples using reverse transcription quantitative polymerase chain reaction. In the gene expression profiling data, CRISP3 was significantly upregulated and had certain proportions on the discriminated principal component of significantly expressed gene sample data. RT-qPCR analysis revealed CRISP3 was significantly upregulated in MM. Therefore, CRISP3 is a potential peripheral blood biomarker for MM.
\end{abstract}

\section{Introduction}

Multiple myeloma (MM) is a neoplastic plasma cell disorder with heterogeneous clinical features (1). The pathogenesis of MMs is a multiple developmental process. However, the underlying molecular mechanism is largely unknown. Monoclonal gammopathy of undetermined significance (MGUS) has an

Correspondence to: Dr Dong Leng, Clinical Laboratory, Beijing Chao-Yang Hospital, Capital Medical University, 8 Worker's Stadium South Road, Beijing 100020, P.R. China

E-mail: lengdong1982@163.com

Key words: multiple myeloma, immunoglobulin heavy chain, cysteine-rich secreted protein 3, octamer-binding protein 2, biomarker incidence of $1 \%$ per year for malignant transformations, and is one of the primary causes of MM. Chromosome translocations or hyperdiploidly frequently occur in MM and MGUS, particularly in immunoglobulin heavy chain (IgH) translocation (2). IgH translocation often results in the juxtaposition of oncogenes to the IgH promoter, thereby causing the overexpression of oncogenes including cyclin D (CCND), V-Maf avian musculoaponeurotic fibrosarcoma, Multiple myeloma SET domain-containing protein and fibroblast growth factor receptor (3-5). A previous study has indicated that transcription factor Octamer-binding protein 2 (Oct2), also known as POU class 2 homeobox 2, may activate the IgH gene enhancer and consequently enhance $\operatorname{IgH}$ gene expression by directly binding to the consensus octamer site ATGCAAAT (4). Notably, CRISP3encoding Cysteine-rich secreted protein 3 (CRISP3) also possesses the ATGCAAAT sequence, and has been demonstrated to be activated by Oct2 in mouse B cells (6-8). This suggests that the deregulation of CRISP3 may contribute to the pathogenesis of MM, and is a novel potential diagnostic or therapeutic biomarker for MM.

Gene expression profiling is a powerful way of understanding the molecular basis of diseases, and has been applied to explore the unique gene expression patterns and distinctly expressed genes of MM from different samples including peripheral blood, bone marrow and isolated cells (9-11). A number of potential biomarkers pertaining to disease classifications and therapy responses have been identified (5). The transcriptional profiling of peripheral blood from patients with MM has provided not only genetic alterations, but also useful diagnostic and prognostic information. However, independent studies (9-11) based on different working platforms have only provided fragmented genetic data; and the peripheral blood environment is usually complicated and affected by numerous factors. Therefore, in order to be precise, the integrated analysis of the gene expression patterns of peripheral blood of $\mathrm{MM}$ is essential for accurate diagnosis, patient stratification and prognosis.

Meta-analysis-based computational methods have provided general information from different studies, and have been successfully utilized in certain genetic disorders (12). In the present study, the transcriptional changes of MM peripheral blood from the super array data derived from different 
studies (9-11) were analyzed, and the principal components from this data were extracted. These data were additionally analyzed by Bayesian probit regression modeling. Key discriminate genes were additionally grouped and annotated. As CRISP3 was identified to be upregulated in other types of cancer, particularly in prostate cancer (PC), the microarray data of PC human peripheral blood samples and the corresponding controls were obtained and re-normalized to the super array data of MM peripheral blood and the control. The selected Oct2, Alpha-1B-glycoprotein (A1GB), CCND2 and CRISP3were validated by reverse transcription quantitative polymerase chain reaction (RT-qPCR) in MM and healthy control peripheral blood samples. These theoretical and experimental data analyses may provide useful indications of the process of MM pathogenesis, and assist in the diagnosis and prognosis determination of MM.

\section{Materials and methods}

In silico studies of gene expression profiling data Gene expression datasets. All gene expression datasets were searched and downloaded from the Gene Expression Omnibus (GEO) repository of the National Center for Biotechnology Information (NCBI) web (http://www.ncbi.nlm.nih.gov/gds/, 3rd August 2015) with the keyword 'multiple myeloma' through the searching engine (13). The inclusion criteria for the present study were datasets comprising Homo sapiens blood sample types and the commonly used microarray platforms [including Affymetrix (Thermo Fisher Scientific, Inc., Waltham, MA, USA); Agilent (Agilent Technologies, Inc., Santa Clara, CA, USA); and Illumina (Illumina, Inc., San Diego, CA, USA)], including different versions of Affymetrix products (Thermo Fisher Scientific, Inc.), including HG-U133_Plus_2, HuGene-1_0-st and HG_U95Av2.

Data preprocess. The series matrix file (txt version) of each dataset was used directly in the data analysis. For the purposes of easier plotting and reducing the level of variation, all measurements were $\log _{2}$-transformed in advance. Probe names of each data were replaced with their corresponding official gene symbols (gene names), and the original sample names were renamed according to their status for ease. As different versions of Affymetrix platforms possess different numbers of probes, probes were designed specifically according to the initial experimental objectives of the analyzed studies (9-11), in the attempt to measure corresponding genes. In the present study, different microarray data were combined via selecting common genes together with the corresponding measurements within different datasets in an attempt to get more precise discriminated genes from MM and the healthy control whole blood samples. Median values were used when one gene was repeatedly detected by $>1$ probe.

Normalization. In order to allow the data of different studies to be comparable from each other, the cross-platform normalization (XPN) method (14) was performed on preprocessed data. $\mathrm{R}$ commands, together with the Bioconductor package CrOss-platform Normalization in R (CONOR_1.0.1) (the package is no longer available from the webpage http://alborz. sdsu.edu/conor, but was originally downloaded 9th March, 2014 and is now available on request from the corresponding author), were utilized for data normalization $(14,15)$. During the normalization process, the sum of the squared Euclidean distance converged to a local minimum, and data were iteratively clustered. This procedure would not only eliminate systematic errors among each individual data, but would also maintain the complete biological information to ensure that all information of gene expression data was not affected by normalization.

General expression pattern prediction. Gene expression of $\mathrm{MM}$ are affected by environmental and genetic factors (16). The genetic features of the disease states is represented by the majority of specimens in the form of gene expression patterns, and has been revealed by a previous study investigating the genetic lesions of MM (17). A two-way clustering method (based on hierarchical clustering algorithms that processed by the Euclidean distance similarity metric and the complete linkage clustering using the Gene Clustering 3.0 and Java TreeView software; the open source software is available from the website, http://bonsai.hgc. $\mathrm{jp} / \sim$ mdehoon/software/cluster/software.htm) was used to determine differentially expressed genes that distinguished between MM and the healthy controls. Distinctive samples that could not be clustered into common conditions were not considered for additional analyses. In order to identify the clearly distinguished expression pattern between normal and MM samples, a student's t-test was also performed on the combined microarray data. Genes with $\mathrm{P}<0.05$ were considered to be differentially expressed.

Principal component extraction and building of the Bayesian probitregression model. Gene expression data possess multiple dimensional properties. Each gene may have interactions with others, and this interaction association can be worked out by data mining. In the present study, the principal components of genes from the MM peripheral blood data with significantly expressed genes were extracted, and the expression patterns were characterized. Each gene has a loading coefficient with each principal component. In addition, Principal components with a cumulative proportion of $85 \%$ were selected for Bayesian probit regression modeling.

Gene enrichment analysis. Significantly expressed annotations of genes were obtained through the online Database for Annotation, Visualization and Integrated Discovery (DAVID) search engine v.6.7 (http://david.abcc.ncifcrf.gov/) $(18,19)$. Genes were classified according to their known biological functions, and gene enrichments of each term were also visualized.

CRISP3 expression among MM control and MM samples, and PC control and PC samples, in whole blood of the Gene Expression Omnibus (GEO) profiles. CRISP3 gene expression data of different disease types were obtained through the GEO profile repository (https://www.ncbi.nlm.nih. gov/geoprofiles/) on 3rd of August 2015. All measurements were normalized by the XPN method, and gene expression of CRISP3 among different sample types was compared across different studies. 
Table I. Primers used in reverse transcription quantitative polymerase chain reaction.

\begin{tabular}{lll}
\hline Gene & \multicolumn{1}{c}{ Forward primer } & \multicolumn{1}{c}{ Reverse primer } \\
\hline CRISP3 & 5'-ACCTTGTGCCAGTTGCCCAGA-3' & 5'-CCCTGACCAACTGATGTTTACAGG-3' \\
Oct2 & 5'-AGGGAAGCCGGCCAGCTACAG-3' & 5'-AGGGGATGGAATTGAGGGGGG-3' \\
A1BG & 5'-CCCAGCACGCCGGCAACTAC-3' & 5'-TCAGCTTTCTGCCACCAGGAGCTC-3' \\
CCND2 & 5'-CAGCACTCTTCCCAGACACACAGTC-3' & 5'-AGGCTATTGAGGAGCACCGCC-3'
\end{tabular}

CRISP3, Cysteine-rich secreted protein 3; Oct2, Octamer-binding protein 2; A1BG, Alpha-1B-glycoprotein; CCND2, cyclin D2.

Table II. Gene expression profile datasets used in data mining.

\begin{tabular}{|c|c|c|c|c|}
\hline $\begin{array}{l}\text { Accession } \\
\text { number }\end{array}$ & Platform & $\begin{array}{c}\text { Total } \\
\text { transcripts }\end{array}$ & $\begin{array}{c}\text { Total } \\
\text { sample number }\end{array}$ & $\begin{array}{c}\text { Normal } \\
\text { sample number }\end{array}$ \\
\hline GSE7116 & Affymetrix Human Genome U133 Plus 2.0 Array & 54,675 & 26 & 5 \\
\hline GSE27838 & Affymetrix Human Genome U133 Plus 2.0 Array & 54,675 & 32 & 16 \\
\hline GSE28107 & Affymetrix Human Genome U133A Array & 22,283 & 6 & 3 \\
\hline
\end{tabular}

\section{Selected gene expression validation}

Ethics statement. The present study was approved by the Ethics Committee of Beijing Chao-Yang Hospital, Capital Medical University (Beijing, China). Written informed consent was obtained from all patients.

Gene expression validation by $R T-q P C R$. Total RNA was extracted from whole blood samples (including 18 healthy controls and 12 patients with MM) by the phenol chloroform method. In brief, $250 \mu 1$ EDTA anti-coagulated blood was mixed with $750 \mu 1$ TRIzol $^{\circledR}$ (Life Technologies; Thermo Fisher Scientific, Inc.) and $1 \mu 1 \mathrm{RNA}$ carrier (Beijing TransGen Biotech Co., Ltd, Beijing, China). Following incubation at room temperature for $5 \mathrm{~min}$, the mixture was added to $200 \mu \mathrm{l}$ chloroform and vortexed rigorously at $200 \mathrm{x}$ g under room temperature for $30 \mathrm{sec}$, followed by incubation at room temperature for $3 \mathrm{~min}$. Subsequent to centrifugation at $12,000 \mathrm{xg}$ for $15 \mathrm{~min}$ at $4^{\circ} \mathrm{C}$, the supernatant (400-500 $\left.\mu \mathrm{l}\right)$ was transferred to an RNase-free tube and incubated with equal volume of isopropanol at room temperature for $10 \mathrm{~min}$. The mixture was centrifuged at $12,000 \mathrm{x} \mathrm{g}$ for $10 \mathrm{~min}$ at $4^{\circ} \mathrm{C}$, and the pellet was washed with RNase-free $75 \%$ ethanol, followed by low speed centrifugation at $8,600 \times \mathrm{g}$ for $5 \mathrm{~min}$ at $4^{\circ} \mathrm{C}$. Following the volatilization of almost all the ethanol, the pellet was mixed with $20 \mu \mathrm{l}$ RNase-free dd $\mathrm{H}_{2} \mathrm{O}$. The $20-\mu \mathrm{l}$ RNA solution was reverse transcribed into cDNA by mixing with the 5- $\mu$ l RT-mix (5X all-in-one RT Master Mix, Abcam, Cambridge, MA, USA) at $25^{\circ} \mathrm{C}$ for $10 \mathrm{~min}, 42^{\circ} \mathrm{C}$ for $15 \mathrm{~min}$ and $85^{\circ} \mathrm{C}$ for $5 \mathrm{~min}$. The template cDNA was diluted to $1: 20$ in $\mathrm{ddH}_{2} \mathrm{O}$. RT-qPCR was performed on ABI7500 in a $20-\mu 1$ reaction volume containing $2 \mu \mathrm{l}$ diluted cDNA, $1 \mu \mathrm{l}$ forward and reverse specific primers, and $10 \mu \mathrm{l}$ qPCR mix (EvaGreen qPCR Master Mix-low ROX; Applied Biological Materials, Inc., Richmond, Canada). The thermocycling conditions were as follows: $50^{\circ} \mathrm{C}$ for $2 \mathrm{~min}, 95^{\circ} \mathrm{C}$ for $10 \mathrm{~min}$, followed by 40 cycles of $95^{\circ} \mathrm{C}$ for $15 \mathrm{sec}, 60^{\circ} \mathrm{C}$ for $30 \mathrm{sec}$ and $72^{\circ} \mathrm{C}$ for
$30 \mathrm{sec}$. The expression level of the target gene was normalized to $\beta$-actin using the $\mathrm{Cq}$ method (20). All specific primers are summarized in Table I.

Statistical analysis. Differentially expressed genes in MM samples compared with normal samples were elucidated by supervised classification using the $\mathrm{R}$ command based software Significance Analysis of Microarray (SAM) 4.0 (http://www-stat.stanford.edu/ tibs/SAM/, software accessed on 10th of September, 2015 supplied by Stanford University, CA, USA) on the sample data with a predicted expression pattern (21). False discovery rates were calculated for each differently expressed gene. The T-statistic method predicted significantly and differently expressed genes, and provided a SAM score for each gene. The higher the SAM scores, the more significant the distinctive gene. Fold changes were also calculated as the average of MM gene expression measurements divided by the average of the healthy control gene expression measurements.

Differences in gene expression measurements from the GEO datasets were assessed using an unpaired Student's t-test by Microsoft Excel software 2007 (Microsoft Corporation, Redmond, WA, USA), and $\mathrm{P}<0.05$ was considered to indicate a statistically significant difference. Supervised classification of samples was analyzed using the T-statistic test by SAM 4.0 software supplied by Stanford University, CA, USA, and a SAM score $<0.05$ was considered to indicate a statistically significant difference. The principle components of the gene expression data were extracted using Principle Component Analysis followed with Bayesian probit regression analysis by pr and arm packages under R command: Principle components with cumulative proportions up to $80 \%$ were considered as sufficient for data description, a probit of principle component $<0.05$ was considered as significant different, and a gene with loading coefficient $>0.8$ was considered as a signature. Changes in gene expression validated by RT-qPCR were 

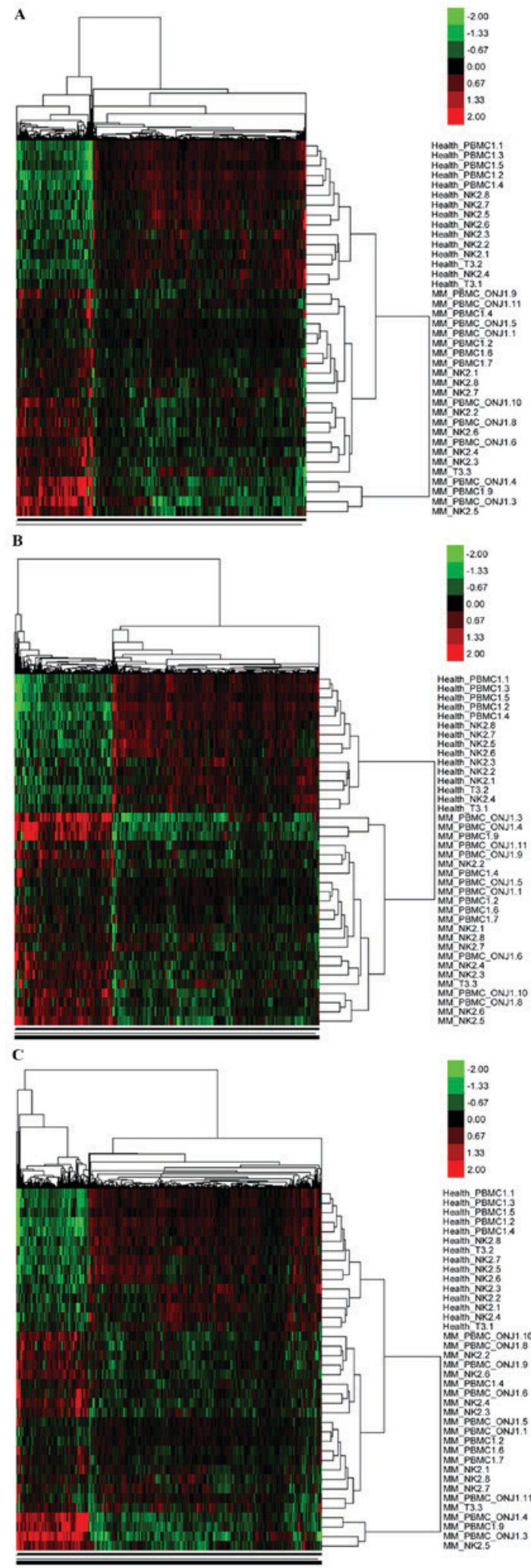

Figure 1. Heat maps of clustering results. Two-way clustering of the gene expression of peripheral blood samples of MM and healthy controls. Red areas are upregulated and green areas are downregulated, whereas black areas are neither up regulated nor down regulated. (A) Gene expression pattern of the merged peripheral blood data. (B) Significantly expressed gene data clustering of peripheral blood samples. (C) Selected gene expression data clustering with higher loading coefficients above CRISP3. MM_PBMC, multiple myeloma peripheral blood mononuclear cell from a patient without osteonecrosis of the jaw; MM_PBMC_ONJ, myeloma peripheral blood mononuclear cell from a patient with osteonecrosis of the jaw; MM_NK, multiple myeloma natural killer cell; MM_T, multiple myeloma T cell; CRISP3, cysteine-rich secreted protein 3.

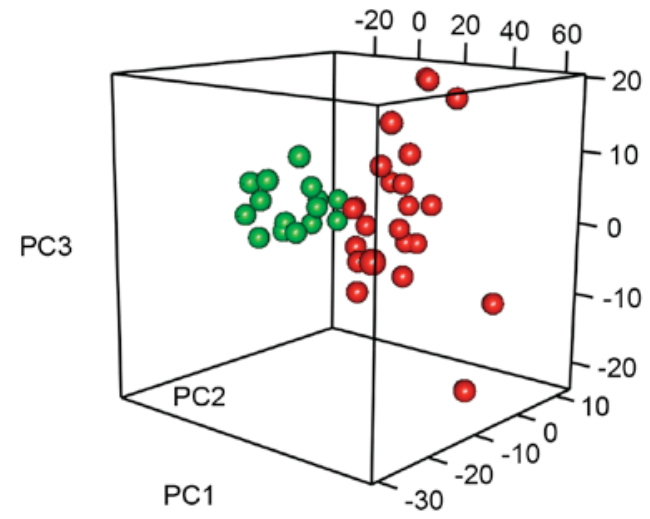

Figure 2. Principal component analysis of peripheral blood data. Principal component analysis of the super array data. Multiple myeloma peripheral blood samples and healthy controls were plotted according to the first three principal components extracted from the super gene array data derived from cross platform normalization. Red dots are MM samples and green dots are healthy controls. PC, prostate cancer.

assessed using an unpaired Student's t-test Prism 5 software (GraphPad Software, Inc., La Jolla, CA, USA), and P $<0.05$ was considered to indicate a statistically significant difference.

\section{Results}

In silico gene expression analysis

Data selection. Through searches using the keyword 'multiple myeloma' in the GEO datasets, 8,918 records were obtained. Among these records, 8,655 were concerning Homo sapiens samples. Within these datasets, 269 intact GSE data contained mostly complete experimental information including summary, overall design, contributors, platforms, sample information and supplementary files. Subsequent to analyzing these published datasets by title, summary, platform, total transcripts, total samples, sample source, control (normal) sample number, control (normal) sample source, data format, dataset contributor and references, 3 human peripheral blood datasets were obtained (Table II). All of these were derived from experiments that were used to compare the gene expression of MM with normal samples.

Preprocessed raw data. The selected gene expression data were compared with each other to produce a common gene list. In the present study, a total of 1,3193 common genes were obtained. In particular, duplicated measurements concerning special genes, including unique genes replaced by different probes and designed by different gene arrays, were combined to calculate their median values.

Super array data. By using the R packages of CONOR, the 3 selected gene expression data were combined together to generate a super array data (data not shown). A total of 48 samples (32 MM and 16 normal samples) from different studies and 13,193 common genes, together with the corresponding measurements, were used to identify the gene expression pattern of MM.

General gene expression pattern. The iterated application of Two-way Clustering and unpaired student's t-test of these 
Table III. Annotation and functional enrichment of differentially expressed genes.

\begin{tabular}{|c|c|c|c|c|c|}
\hline Gene number & Category & Term & Count & $\%$ & FDR \\
\hline 1 & SP_PIR_KEYWORDS & Acetylation & 319 & 1.96 & $3.10 \mathrm{E}-29$ \\
\hline 2 & SP_PIR_KEYWORDS & Phosphoprotein & 658 & 4.04 & $2.32 \mathrm{E}-28$ \\
\hline 3 & SP_PIR_KEYWORDS & Ribosome & 36 & 0.22 & $9.95 \mathrm{E}-20$ \\
\hline 4 & GOTERM_BP_FAT & GO:0006414 translational elongation & 43 & 0.26 & $5.86 \mathrm{E}-19$ \\
\hline 5 & SP_PIR_KEYWORDS & Protein biosynthesis & 55 & 0.34 & $1.70 \mathrm{E}-18$ \\
\hline 6 & KEGG_PATHWAY & hsa03010: Ribosome & 40 & 0.25 & $1.03 \mathrm{E}-17$ \\
\hline 7 & GOTERM_CC_FAT & GO:0022626 cytosolic ribosome & 36 & 0.22 & 7.29E-17 \\
\hline 8 & SP_PIR_KEYWORDS & Ribonucleoprotein & 65 & 0.40 & $1.34 \mathrm{E}-16$ \\
\hline 9 & GOTERM_CC_FAT & GO:0005829 cytosol & 177 & 1.09 & $1.08 \mathrm{E}-15$ \\
\hline 10 & GOTERM_CC_FAT & GO:0044445 cytosolic part & 46 & 0.28 & $1.62 \mathrm{E}-14$ \\
\hline
\end{tabular}

super array data produced the largest general expression pattern of MM, compared with normal samples (Fig. 1A). Among the 48 samples, $23 \mathrm{MM}$ and 15 normal samples demonstrated this pattern; 3,823 differently expressed genes out of 13,193 common genes $(\mathrm{P}<0.05)$ may potentially possess such a gene expression feature.

Significantly and differentially expressed genes. Within the general expression pattern of the super array data, 620 genes were identified as significantly expressed using the SAM software, including 379 up regulated and 241 down regulated genes (Fig. 1B). In addition, genes with a higher coefficient above CRISP3 (for CRISP3, the coefficient was 3.57) conformed to the general expression pattern predicted by unsupervised clustering and supervised classification (Fig. 1C).

Principal component and Bayesian probit regression model. In an attempt to identify more valuable clues from the complicated numeral data, the principal component of the super array for MM peripheral blood was extracted. Each principal component has a proportion that may be used for sample classification, and those components with cumulative proportions $>85 \%$ may provide the primary description of the expression profile of this disease. A total of 19 principal components, with a cumulative proportion of $85.6 \%$, were obtained. These were then used to construct a Bayesian probit regression model. In this regression analysis, only the significant principal component one $(\mathrm{P}<0.05)$ was useful in distinguishing the disease. With this modeling, it was identified that the MM samples and healthy controls were clearly classified by the top 3 principal components (Fig. 2).

Gene annotations. Next, the significantly and differently expressed genes were analyzed using the DAVID software for gene annotation analysis, and 570 biological terms concerning the functions of genes were obtained. The top 10 terms are summarized in Table III. The majority of these significantly and differently expressed genes are involved in gene expression processes, including acetylation, phosphorylation and translation elongation.

CRISP3gene expression across MM normal and MM, and PC normal and $P C$ in whole blood samples of the GEO profiles.

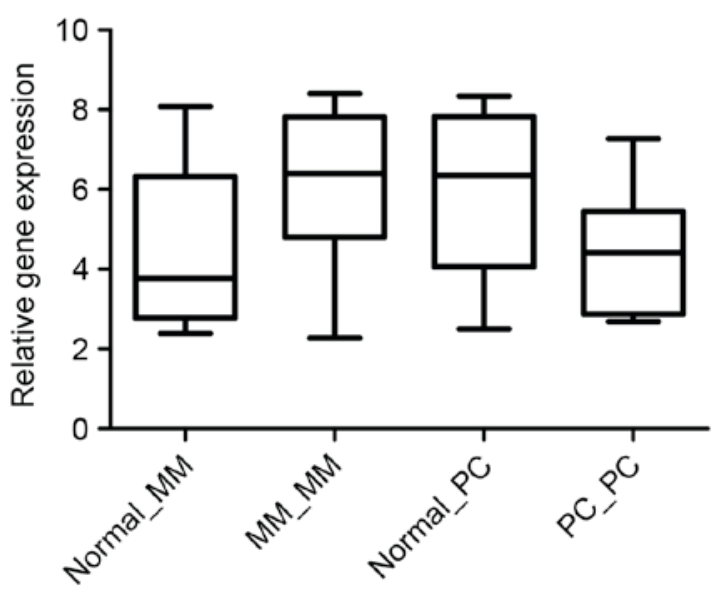

Figure 3. Gene expression level of CRISP3 in whole blood samples of MM normal, MM, PC normal and PC. The expression levels of CRISP3 in different sample types were plotted according to the corresponding gene expression measurements in each study, and all measurements were normalized by XPN method before comparing. Normal_MM, the healthy control peripheral blood samples for multiple myeloma; MM_MM, the multiple myeloma peripheral blood samples; Normal_PC, the healthy control peripheral blood samples for prostate cancer; PC_PC, the prostate cancer peripheral blood samples; CRISP3, cysteine-rich secreted protein 3.

In order to validate the gene expression of crisp3 in other types of cancer, the super array data comparing MM and the corresponding normal control peripheral blood samples were re-normalized to the microarray data comparing $\mathrm{PC}$ and the corresponding normal control. The microarray data of PC and normal peripheral blood samples were downloaded from the GEO profile data of GSE30174 (22). The relative gene expression levels of CRISP3 were plotted according to the normalized gene expression data in each study (Fig. 3). Crisp3 was significantly upregulated $(\mathrm{P}<0.05)$ in $\mathrm{MM}$ compared with the corresponding normal control sample. However, the expression of CRISP3 was not significantly altered between peripheral blood samples of PC and the corresponding controls.

Transcriptional changes of genes validated by RT-qPCR. CRISP3 possesses the Oct2 binding sequence and is activated by Oct 2 in mouse B cells (6-8). A1BG is a secreted protein that exhibits a high affinity with CRISP3, and was identified 


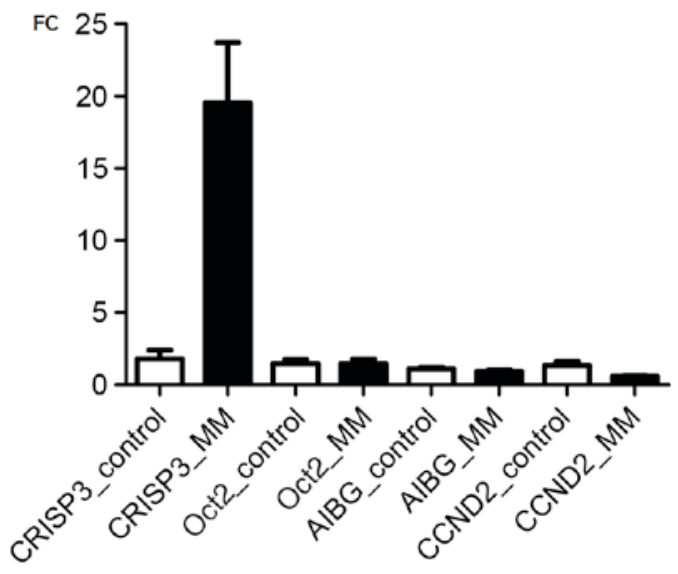

Figure 4. Gene expression validations with RT-qPCR. The RT-qPCR results of CRISP3, Oct2, A1BG and CCND2. Gene expression of genes CRISP3, Oct2, A1BG and CCND2 in MM peripheral blood samples and healthy controls were validated by RT-qPCR. Figures were plotted according to the relative expression levels of target genes normalized to $\beta$-actin housekeeping gene. FC, fold change; RT-qPCR, reverse transcription-quantitative polymerase chain reaction; CRISP3, cysteine-rich secreted protein 3; Oct2, Octamer-binding protein 2; A1BG Alpha-1B-glycoprotein; CCND2, cyclin D2.

to be over expressed in cervical intraepithelial neoplasia (23). CCND2 is a cellular cyclin that has been previously demonstrated to be upregulated in MM (24). In the present study, gene expression of CRISP3, A1BG, CCND2 and Oct2 were additionally validated in whole peripheral blood samples from healthy controls and patients with MM by RT-qPCR (Fig. 4). In comparison to that of peripheral blood samples from healthy controls, CRISP3 was significantly up regulated, with an $\sim 10$-fold increase in peripheral blood samples from patients with $\mathrm{MM}(\mathrm{P}<0.0001)$, while Oct2 was significantly down regulated $(\mathrm{P}<0.05)$ with an $\sim 2.5$-fold decrease. However, there was no significant difference in the expression of A1BG and Oct2 in peripheral blood between patients with MM and normal controls.

\section{Discussion}

IgH translocation leads to the over expression of oncogenes juxtaposed to the IgH enhancers $(4,25,26)$. Although it is generally accepted that IgH translocation occurs frequently either in malignant MM or MGUS (4), little is known about the exact mechanism of MM development. The transcriptional factors Oct2 and B-cell-specific coactivator OBF-1 may activate the expression of IgH by directly binding to the consensus ATGCAAAT octamer, which also exists on the CRISP3 gene $(6,27)$. Previous studies have indicated that the gene expression of CRISP3 was markedly up regulated in prostate carcinoma and closely associated with neoplastic epithelium $(27,28)$. Additionally, CRISP3, together with Transcription regulator ERG and Phosphatase and tensin homolog, defined a molecular subtype of poorest clinical outcomes $(27,28)$, suggesting that CRISP3 is a potential biomarker for prostate cancer. In addition, CRISP 3 expression has been demonstrated to be altered in chronic pancreatitis and oral squamous cell carcinoma (29,30). Furthermore, it was identified that CRISP 3 was activated by Oct 2 in mouse B cells (6-8). Nevertheless, the expression status of CRISP3 and its potential as a peripheral blood biomarker in human MM remains to be determined.

Data of human peripheral blood samples from the GEO repository may be merged together to produce a large sample data, and this super array data possesses a distinguished expression pattern, as observed in previous studies that used tissue samples $(31,32)$. The present study identified that the gene expression of MM peripheral blood also possessed a marked pattern, even in the merged data. Particularly, all MM samples including peripheral blood mononuclear cells, Natural killer cells or even $\mathrm{T}$ cells were clustered together with no bias, compared with healthy controls. This additionally indicates that such expression patterns do not change in peripheral blood by sample type. Notably, the present study revealed that in human peripheral blood samples, CRISP3 was significantly up regulated in the gene expression profiling data from the GEO repository and in the clinical samples. The expression of CRISP3 was significantly up regulated in the unsupervised clustering and supervised classification, indicating that this change is stable. In addition, 19 principal components were extracted from the preprocessed data to produce only 1 marked component by the Bayesian probit regression analysis, and CRISP3 did have certain proportions on this component. Although the expression of CRISP3 was identified to be significantly up regulated in PC tissue in previous studies $(28,33)$, there was no significant difference in CRISP3 expression between PC and normal control peripheral blood samples. These results indicate that CRISP3 may be used as a potential associated biomarker in peripheral blood. In addition, significantly expressed gene enrichment revealed characteristics for MM peripheral blood; for instance, acetylation and phosphorylation. Compared with healthy controls, these biological features of MM peripheral blood samples may partially verify that chromosome change is one of the key events in MM development. However, gene annotation and functional enrichment analysis did not indicate any evidence of CRISP3 participating in the events associated with MM development, which is probably due to the limitation of present studies on gene function. Therefore, future studies on the role of CRISP 3 in chromosome translocation may provide novel insights into the pathogenesis of MM. Furthermore, the expression pattern of CRISP3 in the merged data did not indicate any indications of features for disease progression or for patient prognosis. Additional studies on the role of CRISP3 in different stages of MM will provide novel insight into the precise mechanism of CRISP3 in MM pathogenesis.

Additional experimental validation by RT-qPCR confirmed the observations from the analysis of microarray data. The gene expression of CRISP3 was significantly up regulated in clinical samples from patients with MM patient, while the oncogene CCND2 was down regulated in the MM samples of the present study. Future investigations are required to determine crisp3 expression at the protein level and the mechanism of the up regulation of CRISP 3 in bone marrow cells.

To the best of our knowledge, the present study is the first to investigate the gene expression of human peripheral blood by combing multiple studies in the GEO repository, and has identified distinct expression patterns in the principal component of the merged large sample data, which was additionally validated by clinical peripheral samples. Most importantly, the present study has revealed that CRISP3 is significantly 
over expressed in MM peripheral blood samples. These data suggest that CRISP3 is an associated novel peripheral blood biomarker of MM.

\section{Acknowledgements}

Not applicable.

\section{Funding}

The present study was financially supported by the Beijing Natural Science Foundation (grant nos. 7154198 and 7162069), the National Natural Science Foundation of China (grant nos. 81700061 and 81300044), Beijing Municipal Administration of Hospitals' Youth Programme (grant no. QML20160301) and the open project of Beijing Key Laboratory of Respiratory and Pulmonary Circulation Disorders (grant no. 2014HXFB03).

\section{Availability of data and materials}

All relevant data, including the combined super array data and the CONOR_1.0.1 package, are available upon request from the corresponding author.

\section{Authors' contributions}

Designed the study: DL. Performed the experiments: DL, RM, XH, YW. Analysed the data: DL, RM. Contributed reagents, materials or analysis tools: DL. Wrote the paper: DL, RM. All authors have read and approved the content.

\section{Ethics approval and consent to participate}

All experiments were approved by the Ethics Committee of Beijing Chao-Yang Hospital and written informed consent to participate in this study was obtained from each participant.

\section{Consent for publication}

Written informed consent was obtained from each participant.

\section{Competing interests}

The authors declare that they have no competing interests.

\section{References}

1. Leich E, Weißbach S, Klein HU, Grieb T, Pischimarov J, Stühmer T, Chatterjee M, Steinbrunn T, Langer C, Eilers M, et al: Multiple myeloma is affected by multiple and heterogeneous somatic mutations in adhesion- and receptor tyrosine kinase signaling molecules. Blood Cancer J 3: e102, 2013.

2. Schmidt-Hieber M, Gutiérrez ML, Pérez-Andrés M, Paiva B, Rasillo A, Tabernero MD, Sayagués JM, Lopez A, Bárcena P, Sanchez ML, et al: Cytogenetic profiles in multiple myeloma and monoclonal gammopathy of undetermined significance: A study in highly purified aberrant plasma cells. Haematologica 98: 279-287, 2013.

3. Keats JJ, Maxwell CA, Taylor BJ, Hendzel MJ, Chesi M, Bergsagel PL, Larratt LM, Mant MJ, Reiman T, Belch AR and Pilarski LM: Overexpression of transcripts originating from the MMSET locus characterizes all $\mathrm{t}(4 ; 14)(\mathrm{p} 16 ; \mathrm{q} 32)$-positive multiple myeloma patients. Blood 105: 4060-4069, 2005.
4. Toman I, Loree J, Klimowicz AC, Bahlis N, Lai R, Belch A, Pilarski L and Reiman T: Expression and prognostic significance of Oct 2 and Bob1 in multiple myeloma: Implications for targeted therapeutics. Leuk Lymphoma 52: 659-667, 2011.

5. Szudyszczyrek A, Szczyrek M, Sorokawojtaszko M and Hus M: New prognostic biomarkers in multiple myeloma. Postepy Hig Med Dosw (Online) 70: 811-819, 2016.

6. Pfisterer P, König H, Hess J, Lipowsky G, Haendler B, Schleuning WD and Wirth T: CRISP-3, a protein with homology to plant defense proteins, is expressed in mouse $\mathrm{B}$ cells under the control of Oct2. Mol Cell Biol 16: 6160-6168, 1996.

7. Wirth T, Pfisterer P, Annweiler A, Zwilling S and König H: Molecular principals of Oct2-mediated gene activation in $\mathrm{B}$ cells. Immunobiology 193: 161-170, 1995.

8. Udby L, Cowland JB, Johnsen AH, Sørensen OE, Borregaard N and Kjeldsen L: An ELISA for SGP28/CRISP-3, a cysteine-rich secretory protein in human neutrophils, plasma, and exocrine secretions. J Immunol Methods 263: 43-55, 2002.

9. Raje N, Woo SB, Hande K, Yap JT, Richardson PG, Vallet S, Treister N, Hideshima T, Sheehy N, Chhetri S, et al: Clinical, radiographic, and biochemical characterization of multiple myeloma patients with osteonecrosis of the jaw. Clin Cancer Res 14: 2387-2395, 2008.

10. Garg TK, Szmania SM, Khan JA, Hoering A, Malbrough PA, Moreno-Bost A, Greenway AD, Lingo JD, Li X, Yaccoby S, et al: Highly activated and expanded natural killer cells for multiple myeloma immunotherapy. Haematologica 97: 1348-1356, 2012.

11. Kiaii S, Clear AJ, Ramsay AG, Davies D, Sangaralingam A, Lee A, Calaminici M, Neuberg DS and Gribben JG: Follicular lymphoma cells induce changes in T-cell gene expression and function: Potential impact on survival and risk of transformation. J Clin Oncol 31: 2654-2661, 2013.

12. Rhodes DR, Barrette TR, Rubin MA, Ghosh D and Chinnaiyan AM: Meta-analysis of microarrays: Interstudy validation of gene expression profiles reveals pathway dysregulation in prostate cancer. Cancer Res 62: 4427-4433, 2002.

13. Barrett T and Edgar R: Gene expression omnibus: Microarray data storage, submission, retrieval, and analysis. Methods Enzymol 411: 352-369, 2006.

14. Rudy J and Valafar F: Empirical comparison of cross-platform normalization methods for gene expression data. BMC Bioinformatics 12: 467, 2011.

15. Shabalin AA, Tjelmeland H, Fan C, Perou CM and Nobel AB Merging two gene-expression studies via cross-platform normalization. Bioinformatics 24: 1154-1160, 2008.

16. Shain KH and Dalton WS: Genetic and environmental determinants in multiple myeloma: Implications for therapy. Springer Netherlands 14: 53-82, 2012.

17. Mattioli M, Agnelli L, Fabris S, Baldini L, Morabito F, Bicciato S, Verdelli D, Intini D, Nobili L, Cro L, et al: Gene expression profiling of plasma cell dyscrasias reveals molecular patterns associated with distinct IGH translocations in multiple myeloma. Oncogene 24: 2461-2473, 2005.

18. Huang da W, Sherman BT and Lempicki RA: Systematic and integrative analysis of large gene lists using DAVID bioinformatics resources. Nat Protoc 4: 44-57, 2009.

19. Huang da W, Sherman BT and Lempicki RA: Bioinformatics enrichment tools: Paths toward the comprehensive functional analysis of large gene lists. Nucleic Acids Res 37: 1-13, 2009.

20. Livak KJ and Schmittgen TD: Analysis of relative gene expression data using real-time quantitative PCR and the 2(-Delta Delta C(T)) method. Methods 25: 402-408, 2001.

21. Tusher VG, Tibshirani R and Chu G: Significance analysis of microarrays applied to the ionizing radiation response. Proc Natl Acad Sci USA 98: 5116-5121, 2001.

22. Saligan LN, Hsiao CP, Wang D, Wang XM, St John L, Kaushal A, Citrin D, Barb JJ, Munson PJ and Dionne RA: Upregulation of alpha-synuclein during localized radiation therapy signals the association of cancer-related fatigue with the activation of inflammatory and neuroprotective pathways. Brain Behav Immun 27: 63-70, 2013.

23. Canales NA, Marina VM, Castro JS, Jiménez AA, Mendoza-Hernández G, McCARRON EL, Roman MB and Castro-Romero JI: A1BG and C3 are overexpressed in patients with cervical intraepithelial neoplasia III. Oncol Lett 8: 939-947, 2014.

24. Misiewicz-Krzeminska I, Sarasquete ME, Vicente-Dueñas C, Krzeminski P, Wiktorska K, Quwaider D, Rojas EA, Corral R, Corchete LA, Martin AA, et al: Post-Transcriptional modifications explain the overexpression of CCND2 in multiple myeloma. Blood 124: 2001, 2014. 
25. Scheidereit C, Heguy A and Roeder RG: Identification and purification of a human lymphoid-specific octamer-binding protein (OTF-2) that activates transcription of an immunoglobulin promoter in vitro. Cell 51: 783-793, 1987.

26. Staudt LM, Singh H, Sen R, Wirth T, Sharp PA and Baltimore D: A lymphoid-specific protein binding to the octamer motif of immunoglobulin genes. Nature 323: 640-643, 1986.

27. Al Bashir S, Alshalalfa M, Hegazy SA, Dolph M, Donnelly B and Bismar TA: Cysteine-rich secretory protein 3 (CRISP3), ERG and PTEN define a molecular subtype of prostate cancer with implication to patients' prognosis. J Hematol Oncol 7: 21, 2014.

28. Ribeiro FR, Paulo P, Costa VL, Barros-Silva JD, Ramalho-Carvalho J, Jerónimo C, Henrique R, Lind GE, Skotheim RI, Lothe RA and Teixeira MR: Cysteine-rich secretory protein-3 (CRISP3) is strongly Up-regulated in prostate carcinomas with the TMPRSS2-ERG fusion gene. PLoS One 6: e22317, 2011

29. Liao Q, Kleeff J, Xiao Y, Guweidhi A, Schambony A, Töpfer-Petersen E, Zimmermann A, Büchler MW and Friess H: Preferential expression of cystein-rich secretory protein-3 (CRISP-3) in chronic pancreatitis. Histol Histopathol 18: 425-433, 2003.
30. Ko WC, Sugahara K, Sakuma T, Yen CY, Liu SY, Liaw GA and Shibahara T: Copy number changes of CRISP3 in oral squamous cell carcinoma. Oncol Lett 3: 75-81, 2012.

31. López-Corral L, Corchete LA, Sarasquete ME, Mateos MV, García-Sanz R, Fermiñán E, Lahuerta JJ, Bladé J, Oriol A, Teruel AI, et al: Transcriptome analysis reveals molecular profiles associated with evolving steps of monoclonal gammopathies. Haematologica 99: 1365-1372, 2014.

32. André T, Meuleman N, Stamatopoulos B, De Bruyn C, Pieters K, Bron D and Lagneaux L: Evidences of early senescence in multiple myeloma bone marrow mesenchymal stromal cells. PLoS One 8: e59756, 2013.

33. Grupp K, Kohl S, Sirma H, Simon R, Steurer S, Becker A, Adam M, Izbicki J, Sauter G, Minner S, et al: Cysteine-rich secretory protein 3 overexpression is linked to a subset of PTEN-deleted ERG fusion-positive prostate cancers with early biochemical recurrence. Mod Pathol 26: 733-742, 2013. 